\title{
Effect of Glycyrrhizic Acid based Inductors to Fungi Pathogen (Fusarium solani L.)
}

\author{
U. M. Shapulatov* and H. H. Kushiev \\ Laboratory of Experimental Biology, Gulistan state University, Gulistan city, Uzbekistan \\ *Corresponding author
}

A B S T R A C T

\section{Keywords \\ Glycyrrhiza glabra L., Glycyrrhizic acid, Benzotriazole, Supramolecular complex, Fusarios, in vitro \\ Article Info \\ Accepted: 07 October 2020 Available Online: 10 November 2020}

Nowadays, a chemical drugs are widely used against fungal diseases in crop agriculture.However, there is an inductors that can replace as an alternative solution on this issues. We studied first time the effect of glycyrrhizinic acid with benzotriazole (GA:BT), aminotrazole (GA:AT) and thiadiazole (GA:T) supromolecular complexes and technical Glycyrrhizic acid with micro element complex (CuproTechGA) to Fusarium fungi growth in laboratoty condition. The results showed that GA:BT (4 mg/l concentration) and GA:AT ( $7.5 \mathrm{ml} / 1$ concentration) acted as an inhibitor forFusarium L. Solanimycelium growth.

\section{Introduction}

Large-scale planting of agricultural crops and some of their varieties also contributes to the development of fungal diseases.This fungi disease is a significantly reducing the quality and productivity of crops. Therefore, it is economically, medico-toxicologically, ecologically important issue to increase the resistance of plants to fungal diseases.Some fungi species are a parasitics, them's live cycle depends to other organism livestock (animals and plants). The Fusarium Solani is one of the species in Fusarium L. fungi family that casing a major disease in cereal crops(1).
Fusarium toxins are esealy contaminated with cereal grains, resulting a harmfull cereal based production(2).

The application of chemical fungicides provides both economical and labor benefit in crop agriculture. However, it can have an impact on human health and the environment. In case, there are a number of benefits to use the inductors in the fight against fungal diseases: 1) Reduce the fungicide and pesticides concentrations in soil and plant; 2) Accelerates the growth and development of the plant; 3) Increases the productivity of the yield. Many references showed that using a 
suitable concentration of inductors provide resilience against fungi by synthesis of phenolic compounds in plants(3).

Our previous researches recommended to use a glycyrrhizinic acid (GA) and its complexe with other compounts as a natural fungicide for enhancing of plant resistance to various fungus diseases(4). The GAhas been extracted from the root of licorice (Glycyrrhiza glabra L. And Glycyrrhiza uralica L.)(5)(6). Glycyrrhizic acid (GA) (20 $\beta$-carboxy-11oxo-30-norolean-12-en-3 $\quad \beta$-il-2-O- $\beta$-Dglucopyranuronosyl- $\alpha$-D-glucopyranoseduronic acid) is a valuable raw material, which is important in the food industry, cosmetology, and other fields(7)(8). Several compounds of GA were chemically synthesized and used for various purposes(9). The GA can be easily formed with other molecules to make a supramolecular compoundcalled a "guest-host" type(10). In the present research, the supramolecular complex of GA with benzotriazole, aminotrazole and thiadiazole, as well CuproTechGAwere investigated as a inhibitor of Fusarium L.Solani fungus.

\section{Materials and Methods}

\section{Pathogen material}

Plant fungi pathogen called Fusarium $L$. solani was used in this study. The Fusarium culture were kept on the potato-dextrose at $4^{\circ} \mathrm{C}$, dark condition. One week old culture was used for futher experiments.

\section{Preparation of the fungal culture}

The MS (Murashige Skoog) (11) containing $0.8 \%(\mathrm{w} / \mathrm{v})$ Bacto agar was prepared in 1 litr volume. The medium then adjusted to $\mathrm{pH}-5.8$ and autocleved at $120^{\circ} \mathrm{C}$. A meantime, threeindoctors: Glycyrrhizic acid:Benzotriazole (GA:BT), Glycyrrhizic
acid:Aminotriazole (GA:AT), Glycyrrhizic acid:Thiadiazole (GA:T) complexes, as well a technical Glycyrrhizic acid with micro element complex (CuproTechGA) at $2 \mathrm{mg} / \mathrm{l}, 4$ $\mathrm{mg} / \mathrm{l}, 7.5 \mathrm{mg} / \mathrm{l}, 10 \mathrm{mg} / \mathrm{l}$ consentration were prepared and cold sterilised with special filters. After then the inductors were added to sterilised MS/agar medium and divided to sterile plastic plates. Experiments were followed according to technical and laboratory protocols.

\section{Growing of pathogen}

Fungi disc $(0.5 \times 0.5 \mathrm{sm}$ diametr $)$ from the stock plate was transferred to experimental mediums, respectively. Plates wereincubated at $25^{\circ} \mathrm{C}$ under L/D 16 hours/8 hours of photoperiod and light intensity of 3000 luxfor approximately one week. Then, the plates were taken a photo in same orientation. At least 3 plate were scored from each sample.

\section{Statistic analysis}

Relative size of pathogene was analysed in Image J programme (Bethesda,Maryland, USA). Three replicates were taken for the experiment. Comparison of means was analysed for statistical significance with a 2sample t-test $(\mathrm{P}<0.001)$.

\section{Results and Discussion}

We have synthesized a number of GA-based supramolecular compounds to investing on the pathogenic fungi inhibition (4). From them we have chosen four supramolecular complexes: (GA:BT), (GA:AT), (GA:T) and technical Glycyrrhizic acid with micro element complex (CuproTechGA). For control, it is used only Bacto agar medium. As clearly shown in Figure-1, the lower enrichment rates of F.Solani growth was obtained in GA:BT to compared than control medium. 
Our data showed that increasing the solution concentration of GA-based complexes suppressed the growth and development of the fungus in all senariuos (Figure-2). In the variant of GK: BT $2 \mathrm{mg}$, the diameter of the layer of fungal mycelium was $116.80 \mathrm{~mm}$, which is a $13.37 \mathrm{~mm}$ smaller than this control $(132.18 \mathrm{~mm})$. It was obserbed $11.6 \%$ decrease in treatment (Figure-2,B). Similar indicators were observed under a same consentration of other complexes.

Higher concentration of GA-based compounds were expected to decrease of the fungus development accordingly. The diameter of the mycelial layer was 114.77 $\mathrm{mm}$ in the GA:BT $4 \mathrm{mg}$ variant, $86.96 \mathrm{~mm}$ in the GA: BT $7.5 \mathrm{mg}$, and $63.21 \mathrm{~mm}$ in the GK: BT $15 \mathrm{mg}$ were observed. The fungus diameter was $68.96 \mathrm{~mm}$ smaller than the control in the high-concentration solution. The growth of fungi has slowed by almost $50 \%$. The results in the GA:AT recorded exactly the results obtained with GA:BT variant. Here, increasingof the solution concentration slowed the growth of the fungus. In concentrations of GA: AT $15 \mathrm{mg}$, the diameter of fungal was decreased to 46.66 $\mathrm{mm}$. At the same time, in GA: BT, this value was $68.96 \mathrm{~mm}$. Hence, the GA:AT supramolecular complex had a little effect on fungal growth compared to GA: BT.

The GA:T treatment also affected to the growing of the fungus whenthe solution concentration increased. In the concentration of $2 \mathrm{mg}$, the diameter of the fungal area was $125.47 \mathrm{~mm}$, while a91.91 $\mathrm{mm}$ value shows in higher concentration (GA: T $15 \mathrm{mg}$ ). As a result, the growth diameter of the fungus was $40.26 \mathrm{~mm}$ less than the control (Figure-2,C).

The effect of CuproTechGAsolution was a weaker compared to other options.In this variant, the diameter of the fungal area was $123.47 \mathrm{~mm}$ when the solution concentration was $2 \mathrm{mg}$, and $109.34 \mathrm{~mm}$ in the $15 \mathrm{mg}$ variant. This showed a $22.83 \mathrm{~mm}$ lower value than the control (Figure-2,D). However, the mycelial growth of the F.solani did not show statistical difference between four treatments.

Fig.1 An example illustration for the mycelial growth of Fusarium L. solani under GA with benzotriazole complexes (GA:BT). (A) control; (B) GA:BT 2mg; (C) GA:BT 4mg; (D)GA:BT 7.5mg; (D)GA:BT $15 \mathrm{mg}$

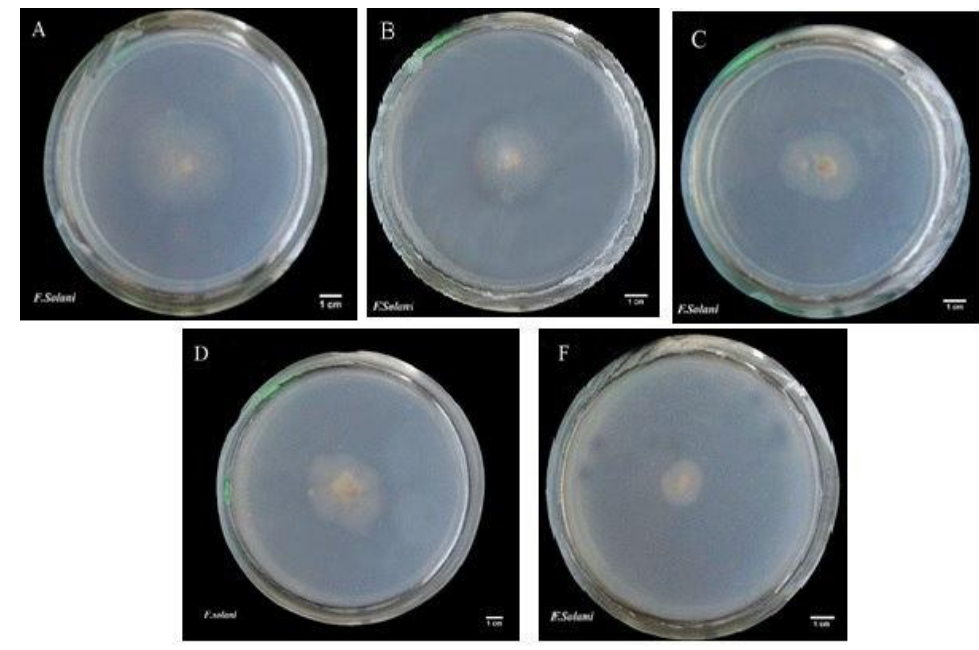


Fig.2 Effects of different concentration of GA-based complexes to mycelial growth of Fusarium L. solani. (A): GA:BT; (B): GA:AT; (C): GA:T; (D) CuproTechGA;

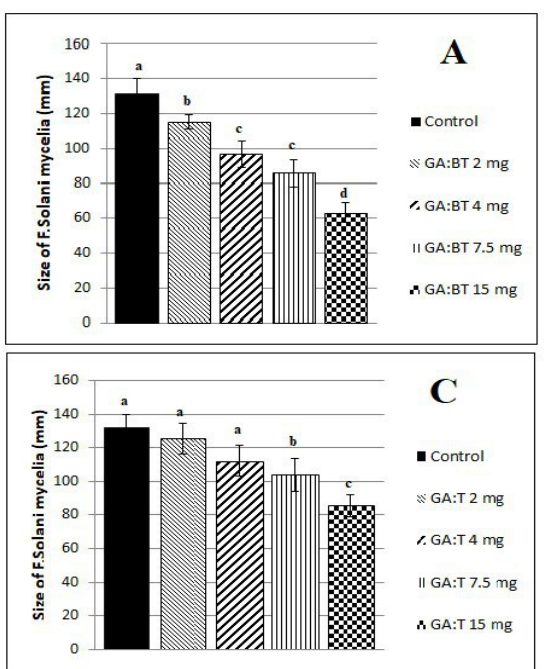

The effect of complexes on the growth of the fungus can be placed in the following sequence: GKBT; GKAT; GK: Tiadiazole; CupriTechGK. Hence, the strongest GK: BT supramolecular complex influenced the growth of the fungus. These type of compounds have also been shown to act as inducers of the germination and resistance of wheat grains during the developmental stages.

The natural compounds do not give a harmful effects on human and animal health or the environment pollution. Therefore, the GA supramolecular complexes as a natural compound allow to use in various directions including agriculture.

In conclusion the glycyrrhizinic acid based supramolecular complexes are highly effective compound can inhibits the fungus species $F$. solani growth and development. The study showed that a $5 \mathrm{mg} / \mathrm{l}$ concentrated solution of the benzotriazole with glycyrrhizinic acid complex and a $7.5 \mathrm{ml} / \mathrm{l}$ concentrated solution of the aminotriazole with glycyrrhizinic acid complex were acted as an inhibitor of the development of all fungal mycelium.

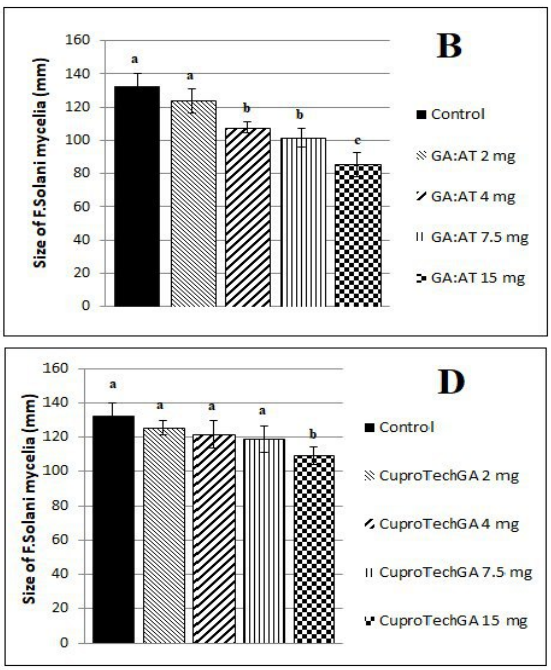

\section{References}

1. A. Bottalico, "Fusarium diseases of cereals: Species complex and related mycotoxin profiles, in Europe," J. Plant Pathol., 1998, doi: 10.4454/jpp.v80i2.807.

2. C. M. Placinta, J. P. F. D'Mello, and A. M. C. Macdonald, "A review of worldwide contamination of cereal grains and animal feed with Fusarium mycotoxins," Anim. Feed Sci. Technol., vol. 78, no. 1, pp. 21-37, 1999, doi: https://doi.org/10.1016/S03778401(98)00278-8.

3. M. Mukhopadhyay and P. Panja, "A novel process for extraction of natural sweetener from licorice (Glycyrrhiza glabra) roots," Sep. Purif. Technol., vol. 63, no. 3, 2008, doi: 10.1016/j.seppur.2008.06.013.

4. D. A. Tulkin and K. Kh. Kushiev, "The Impact of Supramolecular Complex of Glycyrrhizic Acid with Phytohormones on the Wheat (Triticum aestivum L.) Productivity," Int. J. Curr. Microbiol. Appl. Sci., vol. 8, no. 08, 2019, doi: 10.20546/ijcmas.2019.808.017.

5. M. Khanahmadi, R. Ghaffarzadegan, F. Khalighi-Sigaroodi, H. Naghdi Badi, A. 
Mehrafarin, and R. Hajiaghaee, "Optimization of the glycyrrhizic acid extraction from licorice by response surface methodology," Iran. J. Chem. Chem. Eng., vol. 37, no. 1, 2018.

6. E. Karaolul, P. Parlar, H. Parlar, and M. H. Alma, "Enrichment of the Glycyrrhizic Acid from Licorice Roots (Glycyrrhiza glabra L.) by Isoelectric Focused Adsorptive Bubble Chromatography," J. Anal. Methods Chem., vol. 2016, 2016, doi: 10.1155/2016/7201740.

7. H. S. Ju, X. J. Li, B. L. Zhao, Z. W. Han, and W. J. Xin, "Effects of glycyrrhiza flavonoid on lipid peroxidation and active oxygen radicals," Acta Pharm. Sin., vol. 24, no. 11, 1989.

8. Y. Arase et al., "The long term efficacy of glycyrrhizin in chronic hepatitis C patients," Cancer, vol. 79, no. 8, 1997, doi: 10.1002/(SICI)1097-0142(19970415) 79:8<1494::AID-CNCR8>3.0.CO;2-B.
9. O. Y. Selyutina and N. E. Polyakov, "Glycyrrhizic acid as a multifunctional drug carrier - From physicochemical properties to biomedical applications: A modern insight on the ancient drug," International Journal of Pharmaceutics, vol. $559 . \quad 2019$, doi: 10.1016/j.ijpharm.2019.01.047.

10. N. E. Polyakov, V. K. Khan, M. B. Taraban, T. V. Leshina, N. F. Salakhutdinov, and G. A. Tolstikov, "Complexation of lappaconitine with glycyrrhizic acid: Stability and reactivity studies," J. Phys. Chem. B, vol. 109, no. 51, 2005, doi: 10.1021/jp053434v.

11. T. Murashige and F. Skoog, "A Revised Medium for Rapid Growth and Bio Assays with Tobacco Tissue Cultures," Physiol. Plant., vol. 15, no. 3, 1962, doi: 10.1111/j.1399-3054.1962.tb08052.x.

\section{How to cite this article:}

Shapulatov, U. M. and Kushiev, H. H. 2020. Effect of Glycyrrhizic Acid based Inductors to Fungi Pathogen (Fusarium solani L.). Int.J.Curr.Microbiol.App.Sci. 9(11): 826-830.

doi: https://doi.org/10.20546/ijcmas.2020.911.100 